\title{
A CONTRIBUIÇÃO DAS METODOLOGIAS DE APRENDIZAGEM ATIVA PARA A FORMAÇÃO DE ENGENHEIROS DO FUTURO
}

DOI: 10.37702/2175-957X.COBENGE.2021.3629

Jéssica Mendes Jorge - jessicamendes.jmj@gmail.com

University of Brasilia

-Quadra 4961

72455-490 - Brasilia - DF

Andrea Cristina dos Santos - andreasantos@unb.br

Universidade de Brasília

SQN 212

70864-060 - Brasilia - DF

Resumo: As mudanças impulsionadas pelas tecnologias impactam os modelos de negócio, produtos, serviços e sistemas técnicos que exigem profissionais capazes de atuar com equipes multidisciplinares e combinar diferentes áreas de conhecimento para gerarem soluções que resolvam o problema não só no aspecto técnico, mas também considerando o seu impacto econômico, social e ambiental. Deste modo, os engenheiros do futuro devem ser formados em ambientes de aprendizagem que permitam o desenvolvimento de habilidades e competências técnicas, metodológicas, pessoais e sociais. Esses ambientes são compostos pelas metodologias de aprendizagem ativa, que têm como pilares a aprendizagem centrada no aluno, autonomia, reflexão, problematização da realidade, trabalho em equipe, inovação e o professor como facilitador. Para verificar quais habilidades e competências são desenvolvidas pelas metodologias ativas, foi desenvolvido um estudo de caso com uma disciplina da Universidade de Brasília, cujo resultado permitiu verificar a efetividade dessas metodologias.

Palavras-chave: metodologias de aprendizagem ativa; engenheiros do futuro; ambientes de aprendizagem. 


\section{(C) COBENGE \\ 28 a 30 de SETEMBRO \\ A CONTRIBUIÇÃO DAS METODOLOGIAS DE APRENDIZAGEM ATIVA PARA A FORMAÇÃO DE ENGENHEIROS DO FUTURO}

\section{INTRODUÇÃO}

A internet das coisas, realidade aumentada, robôs autônomos, segurança da informação entre outras tecnologias, impulsionam um cenário industrial caracterizado pela integração homem-máquina, digitalização de processos e crescimento vertical e horizontal de toda a cadeia de valor de um setor, cuja oferta de produtos e serviços é cada vez mais assertiva com a necessidade do cliente, por conta do consumo de dados em tempo real (SCHWAB, 2016).

Este cenário fomenta novas modelagens de negócio, produtos, serviços e sistemas técnicos, que exigem dos profissionais de engenharia a capacidade de pensar de forma transdisciplinar e que tenham uma formação que contemple um conjunto de habilidades e competências distribuídas em quatro áreas: metodológica, social, pessoal e técnica (HECKLAU et al., 2016).

Um ponto importante nessa discussão é que essas habilidades não podem ser desenvolvidas em um ambiente de ensino tradicional, mas sim em um sistema que considere o aluno como o centro da aprendizagem e o principal responsável pelo seu próprio desenvolvimento (ABDULWAHED, 2017).

Deste modo, as escolas de engenharia estão reestruturando seus currículos e modelos de ensino, transitando de um escopo puramente técnico, para outro que considera a resolução de problemas do mundo real, interação com diferentes áreas de conhecimento, diferentes contextos e realidades para desenvolver conhecimentos, habilidades, atitudes e valores que formem profissionais com capacidade para compreender problemas e contribuir com soluções para um futuro cada vez mais sustentável (OECD, 2018).

As metodologias utilizadas para incentivar a formação de profissionais com esse escopo de habilidades e competências são denominadas metodologias de aprendizagem ativa. Conforme Araújo et al. (2016), essa abordagem é centrada no aluno e caracterizada por um processo de construção do conhecimento por meio da aplicação de atividades práticas que proporcionam experiências com problemas reais, assim como fomentam a criatividade e aprendizagem de forma dinâmica (MENEKSE et al., 2013).

Contudo, essa mudança na forma de ensinar o aluno tem impacto direto na preparação do professor para planejar disciplinas de aprendizagem ativa, o que foge do contexto em que estão habituados, uma vez que ainda há uma cultura voltada para o ensino tradicional e não há preparo para que estes reproduzam estratégias diferentes daquelas as quais foram submetidos (MEYER et al., 2018).

Deste modo, este artigo busca identificar quais são as habilidades e competências a serem desenvolvidas pelo engenheiro do futuro e, através de um estudo de caso, medir a percepção dos alunos e dos professores no emprego das metodologias de aprendizagem ativa.

\section{HABILIDADES E COMPETÊNCIAS DO ENGENHERIO DO FUTURO}

O processo de formação dos estudantes de engenharia deve considerar o desenvolvimento de um conjunto de habilidades e competências, sendo essas: i) técnicas, uma vez que o conhecimento técnico/disciplinar ainda continuará sendo importante por ser o fator diferencial de cada profissional; ii) cognitivas, relacionadas a forma de pensar, perceber e processar a informação (pensamento crítico, pensamento criativo, aprender a 
aprender, auto regulação); e iii) metodológicas, referentes a capacidade da aplicação do conhecimento na prática, assim como o uso de novas tecnologias (GUERRERO; PALMA; LA ROSA, 2014).

Essas dimensões podem ser percebidas nas Diretrizes Curriculares Nacionais do Curso de Graduação em Engenharia (DCNs de Engenharia), conforme a Resolução no 2 de abril de 2019 do Ministério da Educação (MEC) (Resolução CNE/CES 2/2019, publicada no Diário Oficial da União, Brasília, 26 de abril de 2019, Seção 1, pp. 43 e 44). Nela são apresentadas sete competências que devem ser formadas nos graduandos em engenharia (Quadro 1).

Quadro 1: Competências a serem formadas nos graduandos em engenharia.

Art. 4 $\mathrm{O}$ curso de graduação em Engenharia deve proporcionar aos seus egressos, ao longo da formação, as seguintes competências gerais:

I - Formular e conceber soluções desejáveis de engenharia, analisando e compreendendo os usuários dessas soluções e seu contexto:

\begin{tabular}{c} 
II - Analisar e \\
compreender os \\
fenômenos físicos e \\
químicos por meio de \\
modelos simbólicos, \\
físicos e outros, \\
verificados e validados \\
por experimentação: \\
\hline III - Conceber, projetar e \\
analisar sistemas, \\
produtos (bens e \\
serviços), componentes \\
ou processos:
\end{tabular}
supervisionar e controlar as soluções de Engenharia:

VII - Conhecer e aplicar com ética a legislação e os atos normativos no âmbito do exercício:

VIII - Aprender de forma autônoma e lidar com situações e contextos complexos, atualizandose em relação aos avanços da ciência, da a) ser capaz de utilizar técnicas adequadas de observação, compreensão, registro e análise das necessidades dos usuários e de seus contextos sociais, culturais, legais, ambientais e econômicos;

b) formular, de maneira ampla e sistêmica, questões de engenharia, considerando o usuário e seu contexto, concebendo soluções criativas, bem como o uso de técnicas adequadas;

a) ser capaz de modelar os fenômenos, os sistemas físicos e químicos, utilizando as ferramentas matemáticas, estatísticas, computacionais e de simulação, entre outras.

b) prever os resultados dos sistemas por meio dos modelos;

c) conceber experimentos que gerem resultados reais para o comportamento dos fenômenos e sistemas em estudo.

d) verificar e validar os modelos por meio de técnicas adequadas;

a) ser capaz de conceber e projetar soluções criativas, desejáveis e viáveis, técnica e economicamente, nos contextos em que serão aplicadas;

b) projetar e determinar os parâmetros construtivos e operacionais para as soluções de Engenharia;

c) aplicar conceitos de gestão para planejar, supervisionar, elaborar e coordenar projetos e serviços de Engenharia;

a) ser capaz de aplicar os conceitos de gestão para planejar, supervisionar, elaborar e coordenar a implantação das soluções de Engenharia.

b) estar apto a gerir, tanto a força de trabalho quanto os recursos físicos, no que diz respeito aos materiais e à informação;

c) desenvolver sensibilidade global nas organizações;

d) projetar e desenvolver novas estruturas empreendedoras e soluções inovadoras para os problemas;

e) realizar a avaliação crítico-reflexiva dos impactos das soluções de Engenharia nos contextos social, legal, econômico e ambiental;

a) ser capaz de compreender a legislação, a ética e a responsabilidade profissional e avaliar os impactos das atividades de Engenharia na sociedade e no meio ambiente.

b) atuar sempre respeitando a legislação, e com ética em todas as atividades, zelando para que isto ocorra também no contexto em que estiver atuando;

a) ser capaz de assumir atitude investigativa e autônoma, com vistas à aprendizagem contínua, à produção de novos conhecimentos e ao desenvolvimento de novas tecnologias.

b) aprender a aprender. 
tecnologia e aos desafios

da inovação:

Fonte: Resolução no 2, de 24 de abril de 2019, Capítulo II, Artigo 4o․

Tais competências podem ser observadas no modelo de ensino proposto pelo Instituto de Tecnologia de Massachussetts - MIT. O modelo, denominado CDIO (do inglês conceber, projetar, implementar e operar), busca incentivar o aprendizado de conteúdos fundamentais e avançados da engenharia, em um ambiente com referências claras sobre a função prática do engenheiro em um contexto de aprendizado adequado (Guerreiro, Palma e La Rosa, 2014).

Os principais objetivos do CDIO são: domínio profundo do conhecimento em técnicas fundamentais; liderança na criação e operação de novos produtos, processos e sistemas; compreensão da importância e do impacto estratégico da pesquisa e do desenvolvimento tecnológico na sociedade (ZHANG; LIU, 2009). As competências e habilidades geradas por cada objetivo são (Quadro 2):

Quadro 2: Objetivos do CDIO, projeto proposto pelo MIT

\begin{tabular}{|c|c|}
\hline $\begin{array}{c}\text { Conhecimento } \\
\text { técnico }\end{array}$ & $\begin{array}{c}\text { Conhecimento básico de } \\
\text { ciências. } \\
\text { Conhecimento de áreas } \\
\text { fundamentais da engenharia. } \\
\text { Conhecimento de áreas } \\
\text { fundamentais da engenharia } \\
\text { avançada. }\end{array}$ \\
\hline $\begin{array}{l}\text { Competências } \\
\text { e atributos } \\
\text { pessoais e } \\
\text { profissionais }\end{array}$ & $\begin{array}{l}\text { Raciocínio de engenharia e } \\
\text { resolução de problemas. } \\
\text { Experimentação e descoberta de } \\
\text { conhecimento. } \\
\text { Pensamento sistêmico. } \\
\text { Habilidades e atitudes pessoais. } \\
\text { Habilidades e atitudes } \\
\text { profissionais. }\end{array}$ \\
\hline
\end{tabular}

\begin{tabular}{|c|c|}
\hline Habilidades & \\
interpessoais: & Trabalho em equipe. \\
trabalho em & Comunicação. \\
equipe e & Domínio de uma língua \\
comunicação & estrangeira. \\
\hline
\end{tabular}

Fonte: adaptado de GUERRERO, PALMA e LA ROSA, 2014. Página 835

\begin{tabular}{c|c} 
Conceber, & Contexto externo e social. \\
projetar, & O contexto de negócios e \\
implementar e & empresas. \\
operar sistemas & Conceber. \\
em negócios e & Projetar. \\
contextos sociais & Implementar. \\
& Operar.
\end{tabular}

Outras formas de classificação e definição das habilidades e competências são levantadas por Hecklau et al. (2016), que apresenta quatro dimensões a serem contempladas no processo de formação de um profissional: técnicas, metodológicas, sociais e pessoais (Figura 1 ).

As competências técnicas, compreendem todas as habilidades e conhecimentos técnicos necessários para a realização do trabalho, enquanto as metodológicas estão relacionadas a todas as habilidades e competências para aplicar na prática o conhecimento técnico, tomar decisões e resolver problemas. As sociais englobam todas as habilidades e competências para trabalhar em grupo com atitudes de cooperação, assim como habilidades de comunicação. As competências pessoais incluem as atitudes, motivação e valores sociais dos indivíduos.

E para formar profissionais que provocam e fazem inovação nos ambientes em que estão inseridos e sejam dotados das competências e habilidades do profissional do futuro resumidas por Hecklau et al. (2016) é essencial que durante o processo de formação, os alunos tenham acesso a ambientes e oportunidades que estimulem o questionamento de ideias tradicionais, criação de novas soluções, desenvolvimento de uma cultura de aprendizagem contínua, utilização das informações para resolver problemas reais e 
geração de oportunidades para experimentar as consequências de suas tomadas de decisão (LEMÂITRE, 2018).

Assim, o próximo tópico explora o estado da arte sobre as metodologias que fomentam modelos de educação em engenharia aplicados com vistas ao desenvolvimento de habilidades e competências em um perfil de engenheiro para atender a demanda do mercado.

Figura 1: Habilidades e competências do profissional do futuro.

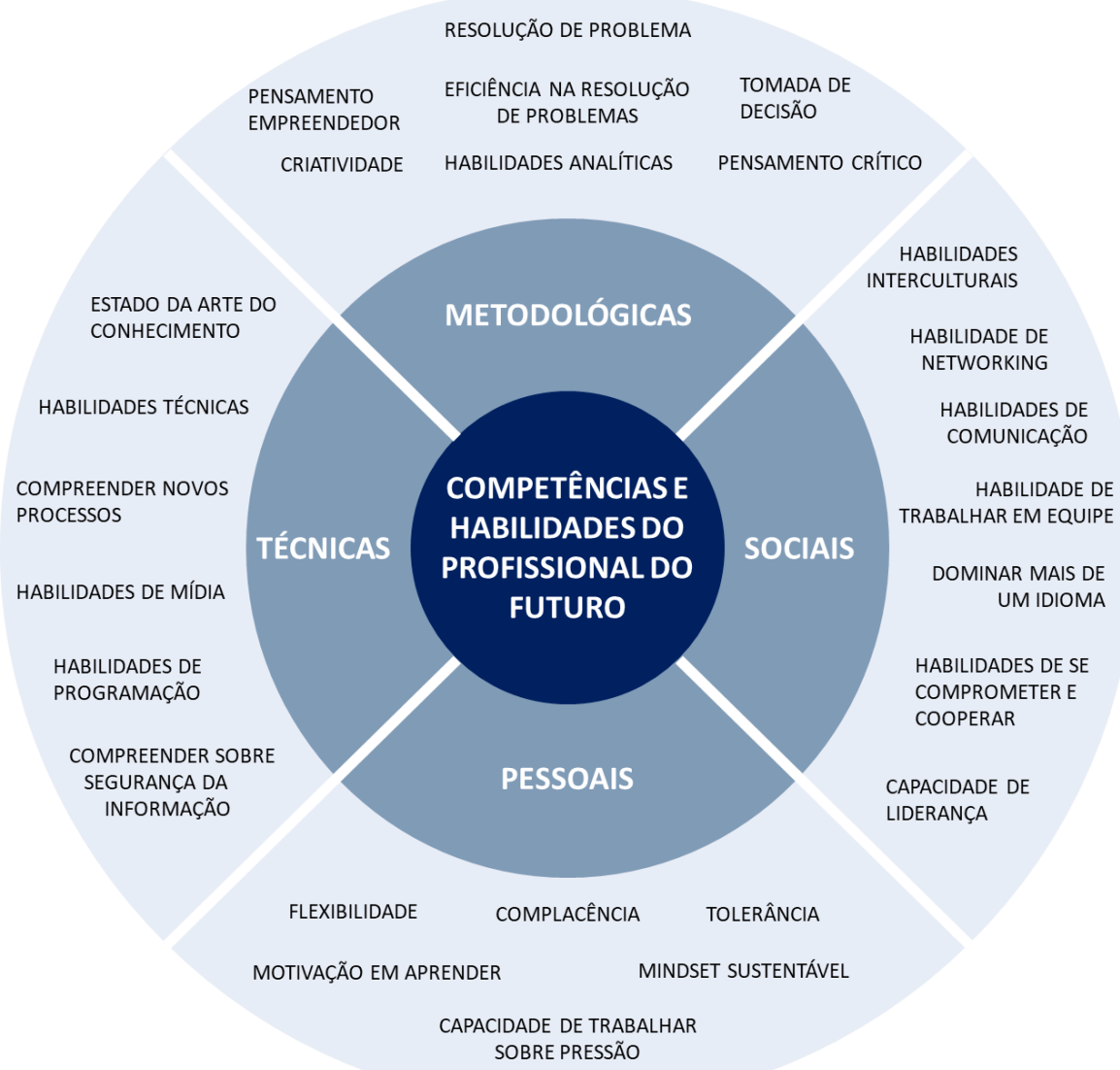

Fonte: Jorge e Santos, 2019.

\subsection{Metodologias de aprendizagem ativa}

As metodologias de aprendizagem ativa são caracterizadas por ambientes que estimulam a postura ativa dos alunos, em um processo no qual estes assimilam o material que está sendo ensinado e não apenas absorvem a informação de forma passiva. Trata-se da transferência de responsabilidade para o estudante, sendo que o professor deixa de ter o papel de principal responsável pela aprendizagem do aluno e passa a ter o papel de guia (TELENKO et al., 2016).

Os métodos de aprendizagem ativa são fundamentados em princípios educacionais e em resultados de pesquisa cognitiva que comprovam que a aprendizagem é um processo dinâmico de construção do conhecimento, cuja participação ativa dos alunos é o elemento fundamental para criar experiências de aprendizagem no nível cognitivo, psicomotor e afetivo, contando com abordagens que envolvem a integração entre a teoria e a prática com situações reais, a fim de desenvolver habilidades e competências que serão requeridas dos estudantes quando forem atuar profissionalmente (ARAÚJO et al., 2016; MENEKSE et al., 2013; RIBEIRO, 2008). 
Assim, o diferencial da aprendizagem ativa está no fato de ter o aluno como o protagonista de um processo de aprendizagem que além da formação técnica e cognitiva, proporciona experiências para a aplicação do conhecimento em situações práticas (DIESEL; BALDEZ; MARTINS, 2017). Os pilares das metodologias de aprendizagem ativa são: aprendizagem centrada no aluno, autonomia, reflexão, problematização da realidade, trabalho em equipe, inovação, professor como facilitador.

Uma forma de provocar uma atuação mais ativa dos alunos em sala de aula e desenvolver a autonomia é utilizando abordagens de problematização da realidade e reflexão, sendo os primeiros pilares das metodologias ativas. Neste cenário papel do professor consiste em instigar a vontade de aprender por meio da problematização dos conteúdos, apresentando-os em contextos de situações reais, fomentando o desenvolvimento de um pensamento orientado a como aplicar e utilizar o conteúdo para compreensão e solução de situações práticas (DIESEL; BALDEZ; MARTINS, 2017).

Em sequência aos pilares, tem-se o trabalho em equipe, derivado da dinâmica aplicada pelas metodologias ativas, que abrange o espaço da sala de aula permitindo mais iteração entre os mesmos, uma vez que a problematização e reflexão sobre situações exige a discussão de ideias, confronto de opiniões, planejamento e tomada de decisões que considerem todos os atores envolvidos (SALINAS-NAVARRO; GARAY-RONDERO, 2019).

O pilar de inovação está relacionado com a capacidade dos docentes em inovar em relação aos métodos de ensino, assim como a capacidade dos alunos em pensar em soluções e aplicar formas inovadoras para encarar as situações problema, estando preparados para contribuir com novos negócios (OECD, 2018).

Por último, tem-se o pilar do professor, que sai do centro do processo de aprendizagem para ter um papel transversal no ensino, guiando o aluno para atingir todos os níveis de aprendizagem cognitiva, afetiva e psicomotora, tendo significado contextualizado e significativo para o estudante.

Essa não é uma tarefa trivial e exige mais esforço do professor, que deve estar em constante renovação dos seus métodos de ensino, além de se reprogramar no sentido de como e quando intervir nas discursões e trabalhos em grupo na sala de aula, como manter a construção simultânea de conhecimentos e como contribuir com a autonomia dos alunos (IGLESIAS; PAZIN-FILHO, 2014).

\subsection{Métodos de aprendizagem ativa}

Uma vez que apresentados os elementos das metodologias de aprendizagem ativa, faz-se necessária a compreensão do estado da arte sobre as formas em que essas metodologias podem ser implementadas. A seguir, são apresentadas as cinco metodologias mais citadas na literatura (JORGE, 2020).

\section{Sala de aula invertida}

O método de sala de aula invertida ou flipped classroom (FC), é caracterizado pela disseminação do conteúdo fora da sala de aula em formato digital e na aula presencial, onde o tempo é destinado para o desenvolvimento de atividades interativas (MASON; SHUMAN; COOK, 2013).

Sua vantagem está na facilitação da interação entre estudantes e professores, proporcionando uma aprendizagem diferenciada por meio da inversão de eventos convencionais dentro e fora da sala de aula, sendo esses apoiados com tecnologias digitais (LAGE; PLATT, 2000).

\section{Aprendizagem hibrida}


O Blended classroom ou Blended Learning (BL), traduzido como aprendizagem híbrida, consiste em instruir o conteúdo por meio da combinação entre o tempo em sala de aula com o tempo de instrução virtual (PORTER et al., 2014).

Além dessa definição, também são encontradas na literatura, referências que definem a aprendizagem combinada como uma junção de modalidades e métodos de ensino, que podem ser aplicados por meio de várias combinações (GRAHAM, 2012).

\section{Aprendizagem baseada em problemas}

O método problem-based learning (PBL), traduzido como aprendizagem baseada em problemas, considera que a aprendizagem é aperfeiçoada pela interação social e que é melhorada quando os estudantes são expostos a situações reais (ARAUUJO et al., 2016).

Este método pode ser utilizado em qualquer contexto ou situação de ensino, podendo oferecer respostas satisfatórias a problemas que tenham consistência com os problemas do mundo real, os quais supostamente irão resolver em suas vidas profissionais (HELMI; MOHD-YUSOF; PHANG, 2016).

\section{Aprendizagem em equipe liderada por pares}

$\mathrm{Na}$ aprendizagem em equipe liderada por pares - PLTL (do inglês peer-led team learning), grupos de aproximadamente oito estudantes são guiados pelos pares, ou seja, mentores treinados para, de forma colaborativa, auxiliar os alunos na resolução de um problema, desafio ou questão apresentada pelo professor em workshops estruturados.

Os mentores são alunos que concluíram a disciplina recentemente, tiveram um bom desempenho e manifestaram interesse em ajudar os outros no processo de aprendizagem, aptidão com o conteúdo e habilidade de comunicação (SNYDER et al., 2016).

\section{Aprendizagem baseada em jogo}

Conforme a revisão da literatura apresentada por Qian e Clark (2016), a aprendizagem baseada em jogos, ou game-based learning (GBL) é caracterizada por um cenário no qual a aplicação de jogos com determinados conteúdos, ambientes e desafios para a solução de problemas, proporciona aos alunos sensações de conquistas e aprimoramento da aquisição de conhecimentos e habilidades desejados.

\subsection{Competências desenvolvidas pelas metodologias de aprendizagem ativa}

Tendo em vista as características das metodologias de aprendizagem ativa apresentadas e as habilidades e competências que devem ser desenvolvidas, conforme Hecklau et al (2017), o Quadro 4 resume 29 habilidades e competências a serem desenvolvidas nos graduandos dos cursos de engenharia.

Neste quadro também foram relacionadas, de forma arbitrária com base na revisão da literatura, quais habilidades e competências do futuro são desenvolvidas por cada metodologia. Contudo, podem ser feitas as seguintes perguntas: de fato essas habilidades são desenvolvidas em contextos reais de aplicação das metodologias?

Em busca de responder estes questionamentos, foi realizado um estudo de caso para identificar quais habilidades e competências são desenvolvidas com a aplicação de disciplinas que utilizam metodologias de aprendizagem ativa.

Quadro 3: Análise das habilidades e competências desenvolvidas pelas metodologias de aprendizagem ativa.

\begin{tabular}{l|l|l|}
\hline DESCRIÇÃO DA & ID & HABILIDADES E \\
CLASSE & COMPETÊNCIAS
\end{tabular}

METODOLOGIAS ATIVAS

\begin{tabular}{l|l|l|l|l} 
FC & PBL & PLTL & GBL & BC
\end{tabular} 


\begin{tabular}{|c|c|c|c|c|c|c|c|c|}
\hline \multirow{9}{*}{ 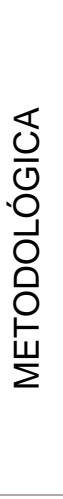 } & \multirow{9}{*}{$\begin{array}{l}\text { Relaciona as } \\
\text { habilidades e } \\
\text { competências } \\
\text { para a resolução } \\
\text { de problemas e } \\
\text { tomada de } \\
\text { decisão. }\end{array}$} & M1 & $\begin{array}{l}\text { Resolução de } \\
\text { problemas }\end{array}$ & & $\mathrm{x}$ & $x$ & $x$ & \\
\hline & & M2 & $\begin{array}{l}\text { Pensamento } \\
\text { empreendedor }\end{array}$ & & $x$ & & $\mathrm{x}$ & \\
\hline & & M3 & $\begin{array}{l}\text { Eficiência na resolução } \\
\text { de problemas }\end{array}$ & & $\mathrm{x}$ & $\mathrm{x}$ & $\mathrm{x}$ & \\
\hline & & M4 & Tomada de decisão & & $x$ & $x$ & $x$ & \\
\hline & & M5 & Criatividade & & $x$ & $x$ & $x$ & \\
\hline & & M6 & Habilidades analíticas & & $x$ & $x$ & & \\
\hline & & M7 & Habilidades de pesquisa & & $x$ & $x$ & & \\
\hline & & M8 & Pensamento crítico & & $\mathrm{x}$ & $\mathrm{x}$ & $\mathrm{x}$ & \\
\hline & & M9 & Pensamento sistêmico & & $\mathrm{x}$ & & & \\
\hline \multirow{6}{*}{ 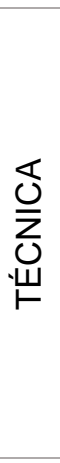 } & \multirow{6}{*}{$\begin{array}{l}\text { Abrange as } \\
\text { habilidades e } \\
\text { conhecimentos } \\
\text { relacionados ao } \\
\text { trabalho. }\end{array}$} & $\mathrm{T} 10$ & $\begin{array}{l}\text { Estado da arte do } \\
\text { conhecimento }\end{array}$ & & $x$ & $x$ & $x$ & \\
\hline & & $\mathrm{T} 11$ & Habilidades técnicas & & $x$ & $x$ & $\mathrm{x}$ & \\
\hline & & $\mathrm{T} 12$ & $\begin{array}{l}\text { Compreender novos } \\
\text { processos }\end{array}$ & & $\mathrm{x}$ & & $\mathrm{x}$ & \\
\hline & & $\mathrm{T} 13$ & Habilidades de mídia & & $x$ & & $x$ & \\
\hline & & $\mathrm{T} 14$ & $\begin{array}{l}\text { Habilidades de } \\
\text { programação }\end{array}$ & & $x$ & & & \\
\hline & & $\mathrm{T} 15$ & $\begin{array}{l}\text { Compreender sobre } \\
\text { segurança da } \\
\text { informação }\end{array}$ & & $x$ & & & \\
\hline \multirow{6}{*}{$\begin{array}{l}\frac{1}{\delta} \\
\text { 仓 } \\
\text { 心 } \\
\text { வ }\end{array}$} & \multirow{6}{*}{$\begin{array}{l}\text { Contempla os } \\
\text { valores sociais, } \\
\text { motivações e } \\
\text { atitudes } \\
\text { individuais. }\end{array}$} & P16 & Flexibilidade & $x$ & $x$ & & $x$ & $\mathrm{x}$ \\
\hline & & P17 & Complacência & $x$ & $x$ & & $x$ & \\
\hline & & P18 & Tolerância & $\mathrm{x}$ & $\mathrm{x}$ & & $\mathrm{x}$ & \\
\hline & & P19 & Motivação em aprender & $\mathrm{x}$ & $\mathrm{x}$ & $x$ & $\mathrm{x}$ & \\
\hline & & P20 & Mindset sustentável & & $\mathrm{x}$ & & & \\
\hline & & P21 & $\begin{array}{l}\text { Capacidade de trabalhar } \\
\text { sobre pressão }\end{array}$ & & $\mathrm{x}$ & & $x$ & \\
\hline \multirow{8}{*}{ 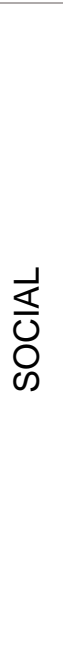 } & \multirow{8}{*}{$\begin{array}{l}\text { Abrange todas as } \\
\text { habilidades, } \\
\text { competências e } \\
\text { atitudes para } \\
\text { cooperar e } \\
\text { comunicar com } \\
\text { outros. }\end{array}$} & S22 & $\begin{array}{l}\text { Capacidade de } \\
\text { liderança }\end{array}$ & & $\mathrm{x}$ & & & \\
\hline & & $\mathrm{S} 23$ & $\begin{array}{l}\text { Habilidades de se } \\
\text { comprometer e cooperar }\end{array}$ & $x$ & $\mathrm{x}$ & $x$ & $\mathrm{x}$ & \\
\hline & & $\mathrm{S} 24$ & $\begin{array}{l}\text { Dominar mais de um } \\
\text { idioma }\end{array}$ & & & & $x$ & \\
\hline & & S25 & $\begin{array}{l}\text { Habilidade de trabalhar } \\
\text { em equipe }\end{array}$ & & $\mathrm{x}$ & $\mathrm{x}$ & $x$ & \\
\hline & & S26 & $\begin{array}{l}\text { Habilidade de } \\
\text { comunicação }\end{array}$ & & $\mathrm{x}$ & $\mathrm{x}$ & $\mathrm{x}$ & \\
\hline & & S27 & $\begin{array}{l}\text { Habilidade de } \\
\text { networking }\end{array}$ & & $\mathrm{x}$ & $x$ & $x$ & \\
\hline & & $\mathrm{S} 28$ & $\begin{array}{l}\text { Habilidades } \\
\text { interculturais }\end{array}$ & & $\mathrm{x}$ & $x$ & $\mathrm{x}$ & \\
\hline & & S29 & $\begin{array}{l}\text { Habilidade de transferir } \\
\text { conhecimento }\end{array}$ & & $x$ & $\mathrm{x}$ & & \\
\hline
\end{tabular}

Fonte: JORGE, 2020.

\subsection{Estudo de caso: Metodologias na prática}

A metodologia utilizada para o desenvolvimento do estudo de caso foi planejada conforme o modelo de Miguel (2007), que é composto por seis etapas: definir uma estrutura conceitual teórica, planejar os casos, conduzir o teste piloto, coletar os dados, analisar os dados e gerar relatório. Por meio dessas etapas, o estudo tem como objetivo verificar de forma prática, quais habilidades e competências o professor identificou que foram desenvolvidas a partir de uma disciplina com o uso de metodologias de aprendizagem ativa, e comparar a percepção do professor com a dos alunos. 
A disciplina utilizada como objeto do estudo de caso, Projeto em Sistemas de Produção 6 (PSP6), faz parte do curso de Engenharia de Produção da Universidade de Brasília (UnB) e aplica a metodologia de aprendizagem ativa baseada em problemas, tendo como ementa: o desenvolvimento de projeto em grupo com aplicação dos conceitos apresentados na disciplina âncora de Engenharia de Produto. Os alunos formam grupos, que se mantém durante todo o semestre e cada um escolhe uma situação-problema para desenvolver uma solução, utilizando a metodologia de desenvolvimento de produto. No ato da conclusão da disciplina, cada grupo deve apresentar um protótipo físico de sua solução.

Para efetuar a coleta dos dados foram elaborados dois formulários com base no Quadro 4, um destinado ao professor e outro destinado ao aluno. O intuito da aplicação foi verificar quais habilidades e competências são desenvolvidas levando em consideração os dois pontos de vista. Desta forma, os formulários tinham como alternativa de resposta (Quadro 5):

Quadro 4: alternativas de resposta dos formulários.

\begin{tabular}{|l|c|}
\hline Alternativa & Valor \\
\hline A habilidade foi muito bem desenvolvida. & 5 \\
\hline A habilidade foi parcialmente desenvolvida. & 4 \\
\hline Não consigo identificar se essa habilidade foi desenvolvida. & 3 \\
\hline A habilidade foi mal desenvolvida. & 2 \\
\hline A habilidade não foi desenvolvida. & 1 \\
\hline
\end{tabular}

Fonte: elaboração própria.

A aplicação do formulário contou com a resposta do professor e de 46 alunos da disciplina. Na visão do professor, a dimensão metodológica teve cinco habilidades muito bem desenvolvidas e quatro parcialmente desenvolvidas. $\mathrm{Na}$ dimensão técnica duas habilidades foram muito bem desenvolvidas, duas parcialmente desenvolvidas e uma não foi desenvolvida por não fazer parte do escopo da disciplina (T14 - habilidade de programação).

$\mathrm{Na}$ dimensão pessoal, o professor julgou que todas as habilidades foram parcialmente desenvolvidas, exceto a habilidade P19 (motivação em aprender) julgada como muito bem desenvolvida. Na dimensão social, todas as habilidades foram apontadas como muito bem desenvolvidas, exceto a habilidade S24 (dominar mais de um idioma), cujo professor apontou que tal habilidade não foi requisitada.

Com base nas respostas obtidas pelos alunos, a dimensão metodológica teve todas as habilidades e competências apontadas como muito bem desenvolvidas, exceto a criatividade e o pensamento crítico (47\%). Na dimensão técnica, os alunos identificaram que o estado da arte do conhecimento, habilidades técnicas e compreensão de novos processos foram muito bem desenvolvidas, em compensação, a habilidade de mídia, habilidade de programação e compreensão sobre a segurança da informação foram apontadas como não desenvolvida por 36\%, 73\% e 52\% dos alunos respectivamente.

Seguindo para a dimensão pessoal, todas as habilidades e competências foram apontadas como muito bem desenvolvidas. Na dimensão social, praticamente todas foram bem desenvolvidas, com exceção do domínio de mais de um idioma, que foi apontado por $52 \%$ dos alunos como não desenvolvido.

A comparação das respostas dos alunos e do professor em relação ao desenvolvimento de habilidades e competências durante a disciplina de PSP6 permite concluir que as expectativas do professor em relação ao desenvolvimento de habilidades e competências são atendidas na dimensão metodológica, superadas na dimensão pessoal 
e foram superestimadas nas dimensões técnica e social, sendo a maior divergência percebida na dimensão técnica.

Assim como observado no gráfico de comparação geral da Figura 2, a disciplina de PSP6 contou o desenvolvimento muito próximo do que era esperado pelo professor, na dimensão metodológica por exemplo, a habilidade M5 (criatividade) foi a que mais se distanciou do esperado pelo professor, que a identificou como muito bem desenvolvida e os alunos a apontaram como parcialmente desenvolvida.

Figura 2: Comparação entre as respostas do professor e dos alunos.

\section{Comparação Específica PSP6 \\ Dimensão Metodológica}

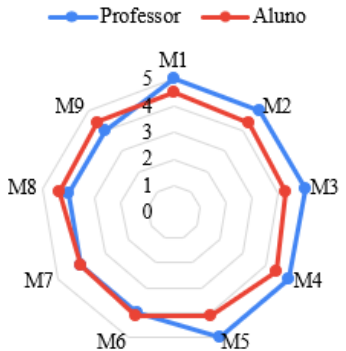

Comparação Específica PSP6

Dimensão Pessoal

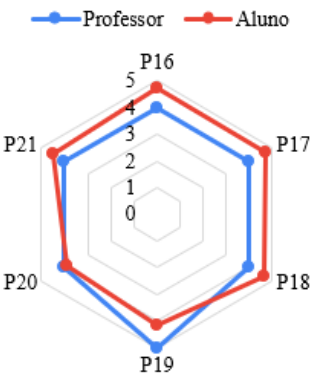

Comparação Específica PSP6 Dimensão Técnica

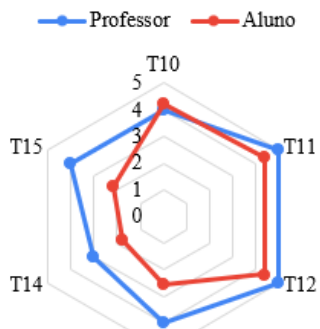

$\mathrm{T} 13$
Comparação Específica PSP6

Dimensão Social
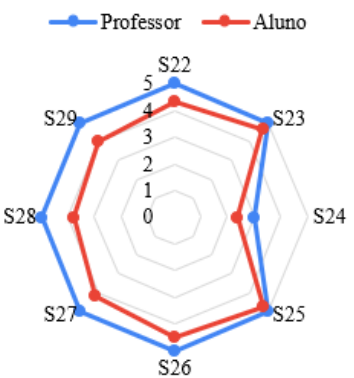

Fonte: JORGE, 2020.

$\mathrm{Na}$ dimensão técnica as expectativas do professor não foram atingidas para as habilidades T13 (habilidades de mídia), T14 (habilidades de programação) e T15 (compreensão sobre segurança da informação). Na dimensão social, nenhuma atividade chegou a atingir de fato o desenvolvimento esperado pelo professor, principalmente a S27 (habilidade de networking), S28 (habilidades interculturais) e S29 (habilidade de transferir conhecimento). Por fim, na dimensão pessoal, as habilidades e competências desenvolvidas superaram a expectativa do professor.

\section{Considerações FINAIS}

As habilidades e competências que compõem o perfil do engenheiro do futuro, podem ser divididas em quatro dimensões: metodológica, técnica, social e pessoal. Cada uma dessas dimensões tem um papel importante para que o aluno se torne um profissional capaz de resolver problemas complexos, com equipes e recursos transdisciplinares, pensando no impacto social, ambiental e econômico da solução proposta.

Para desenvolver esse mindset é necessária a aplicação de metodologias de aprendizagem ativa, que inserem o aluno no centro do processo de aprendizagem e proporcionam o contato com experiências reais, que o colocam a prova em relação a como combinar diferentes áreas de conhecimentos para solucionar um problema. 
O estudo de caso aplicado, permite concluir que há uma contribuição das metodologias ativas no desenvolvimento das habilidades e competências apresentadas na literatura.

Acredita-se que tal estudo possa ser replicado, utilizando um método de medição do conhecimento do aluno antes e depois de cursar a disciplina, assim, seria possível obter um resultado mais preciso sobre a contribuição das metodologias para a formação de engenheiros do futuro.

\section{REFERÊNCIAS}

ABDULWAHED, M. Technology Innovation and Engineering' Education and Entrepreneurship (TIEE) in engineering schools: Novel model for elevating national knowledge based economy and socio-economic sustainable development. Sustainability (Switzerland), v. 9, n. 2, 2017.

ARAÚJO, W. J. DE et al. Aprendizagem por problemas no ensino de Engenharia. Revista Docência do Ensino Superior, v. 6, n. 1, p. 57-90, 2016.

BRASIL. Ministério da Educação. Resolução nํ 2, de 24 de abril de 2019. Institui as Diretrizes Curriculares Nacionais do Curso de Graduação em Engenharia.

DIESEL, A.; BALDEZ, A. L. S.; MARTINS, S. N. Os princípios das metodologias ativas de ensino: uma abordagem teórica Active teaching methodologies principles: a theoretical approach. Revista Thema, v. 14, n. 268, p. 268-288, 2017.

GRAHAM, C. R. Emerging practice and research in blended learning (draft version before print). Handbook of distance education ( $3 \mathrm{rd} \mathrm{ed} \mathrm{.).} \mathrm{Emerging} \mathrm{Practice} \mathrm{and} \mathrm{Research} \mathrm{in}$ Blended Learning, n. 801, p. 333-350, 2012.

GUERRERO, D.; PALMA, M.; LA ROSA, G. Developing Competences in Engineering Students. The Case of Project Management Course. Procedia - Social and Behavioral Sciences, v. 112, n. Iceepsy 2013, p. 832-841, 2014.

HECKLAU, F. et al. Holistic Approach for Human Resource Management in Industry 4.0. Procedia CIRP, v. 54, n. June 2017, p. 1-6, 2016.

HELMI, S. A.; MOHD-YUSOF, K.; PHANG, F. A. Enhancement of team-based problem solving skills in engineering students through cooperative problem-based learning. International Journal of Engineering Education, v. 32, n. 6, p. 2401-2414, 2016.

IGLESIAS, A. G.; PAZIN-FILHO, A. Estr a tégias ino v ador as par a métodos Estra inov adoras para de ensino tr adicionais - aspectos tradicionais g er ais erais. v. 47, n. 3, 2014.

JORGE, Jéssica Mendes. Planejamento sistemático de disciplinas com o uso de aprendizagem ativa para a formação do engenheiro empreendedor. 2020.

Dissertação (Mestrado) - Curso de Pós-Graduação em Sistemas Mecatrônicos. Universidade de Brasília, Brasília, 2020.

JORGE, J. M.; SANTOS, A. C. DOS; PROPOSTA DE UMA ESTRUTURA PARA FORMAÇÃO DO ENGENHEIRO EMPREENDEDOR. XXVI SIMPÓSIO DE ENGENHARIA 
DE PRODUÇÃO, p. 1-12, 2019.

LAGE, M. J.; PLATT, G. Inverting the Classroom: A Gateway to Creating an Inclusive Learning Environment. n. December, 2000.

LEMÂITRE, D. Training Engineers for Innovation. [s.I.] ISTE Ltd, 2018.

MASON, G. S.; SHUMAN, T. R.; COOK, K. E. Comparing the effectiveness of an inverted classroom to a traditional classroom in an upper-division engineering course. IEEE Transactions on Education, v. 56, n. 4, p. 430-435, 2013.

MENEKSE, M. et al. Differentiated overt learning activities for effective instruction in engineering classrooms. Journal of Engineering Education, v. 102, n. 3, p. 346-374, 2013.

MEYER, P. et al. O desafio da mobilização institucional na formação docente. Educação, v. 41, n. 2, p. 250, 2018.

MIGUEL, P. A. C. Estudo de caso na engenharia de produção: estruturação e recomendações para sua condução. Production, v. 17, n. 1, p. 216-229, 2007.

OECD. The future of education and skills Education 2030. p. 23, 2018.

PORTER, W. W. et al. Blended learning in higher education: Institutional adoption and implementation. Computers and Education, v. 75, p. 185-195, 2014.

QIAN, M.; CLARK, K. R. Game-based Learning and 21st century skills: A review of recent research. Computers in Human Behavior, v. 63, p. 50-58, 2016.

RIBEIRO, L. R. DE C. APRENDIZAGEM BASEADA EM PROBLEMAS ( PBL ) NA EDUCAÇÃO EM ENGENHARIA. Revista de Ensino de Engenharia, v. 27, n. 2, p. 23-32, 2008.

SALINAS-NAVARRO, D. E.; GARAY-RONDERO, C. L. Experiential learning in Industrial Engineering education for Digital Transformation. n. August, 2019.

SCHWAB, K. The Fourth Industrial Revolution. Geneva: World Economic Forum, 2016.

SNYDER, J. J. et al. Peer-Led Team Learning Helps Minority Students Succeed. p. 1-7, 2016.

TELENKO, C. et al. Designettes: An Approach to Multidisciplinary Engineering Design Education. Journal of Mechanical Design, Transactions of the ASME, v. 138, n. 2, 2016.

ZHANG, Y.; LIU, J. An Experiment of Computer Curriculum Reform Based on CDIO in Engineering Education. p. 3519-3522, 2009. 


\title{
THE CONTRIBUTION OF ACTIVE LEARNING METHODOLOGIES TO THE TRAINING OF FUTURE ENGINEERS
}

\begin{abstract}
The changes driven by technologies impact the business models, products, services and technical systems, require professionals capable of working with multidisciplinary teams and combining different areas of knowledge to generate solutions that solve the problem not only in the technical aspect but also considering their own economic, social and environmental impact. Thus, the engineers of the future must be trained in learning environments that allow the development of technical, methodological, personal, and social skills and competencies. These environments are composed of active learning methodologies based on student-centered learning, autonomy, reflection, problematizing reality, teamwork, innovation and the teacher as a facilitator. To verify which skills and competencies are developed by the active methodologies, a case study was developed with a discipline from the University of Brasilia and the results allowed to verify the effectiveness of these methodologies.
\end{abstract}

Keywords: active learning methodologies; engineers of the future; learning environments. 